\title{
On the maximum modulus of a polynomial and its polar derivative
}

Ahmad Zireh

Correspondence:

azireh@shahroodut.ac.ir

Department of Mathematics,

Shahrood University of Technology,

Shahrood, Iran

\section{Abstract}

For a polynomial $p(z)$ of degree $n$, having all zeros in $|z| \leq 1$, Jain is shown that

$$
\begin{array}{r}
\max _{|z|=1}\left|D_{\alpha_{t}} \cdots D_{\alpha_{2}} D_{\alpha_{1}} p(z)\right| \geq \frac{n(n-1) \cdots(n-t+1)}{2^{t}} \times[ \\
\left\{\left(\left|\alpha_{1}\right|-1\right) \cdots\left(\left|\alpha_{t}\right|-1\right)\right\} \max _{|z|=1}|p(z)|+ \\
\left.\left\{2^{t}\left(\left|\alpha_{1}\right| \cdots\left|\alpha_{t}\right|\right)-\left\{\left(\left|\alpha_{1}\right|-1\right) \cdots\left(\left|\alpha_{t}\right|-1\right)\right\}\right\} \min _{|z|=1}|p(z)|\right], \\
\left|\alpha_{1}\right| \geq 1, \quad\left|\alpha_{2}\right| \geq 1, \cdots\left|\alpha_{t}\right| \geq 1, \quad(t<n) .
\end{array}
$$

In this paper, the above inequality is extended for the polynomials having all zeros in $|z| \leq k$, where $k \leq 1$. Our result generalizes certain well-known polynomial inequalities.

(2010) Mathematics Subject Classification. Primary 30A10; Secondary 30C10, $30 \mathrm{D} 15$

Keywords: Polar derivative, Polynomial, Inequality, Maximum modulus, Zeros

\section{Introduction and statement of results}

Let $p(z)$ be a polynomial of degree $n$, then according to the well-known Bernstein's inequality [1] on the derivative of a polynomial, we have

$$
\max _{|z|=1}\left|p^{\prime}(z)\right| \leq n \max _{|z|=1}|p(z)| \text {. }
$$

This result is best possible and equality holding for a polynomial that has all zeros at the origin.

If we restrict to the class of polynomials which have all zeros in $|z| \leq 1$, then it has been proved by Turan [2] that

$$
\max _{|z|=1}\left|p^{\prime}(z)\right| \geq \frac{n}{2} \max _{|z|=1}|p(z)|
$$

The inequality (1.2) is sharp and equality holds for a polynomial that has all zeros on $|z|=1$.

As an extension to (1.2), Malik [3] proved that if $p(z)$ has all zeros in $|z| \leq k$, where $k \leq 1$, then 


$$
\max _{|z|=1}\left|p^{\prime}(z)\right| \geq \frac{n}{1+k} \max _{|z|=1}|p(z)|
$$

This result is best possible and equality holds for $p(z)=(z-k)^{n}$.

Aziz and Dawood [4] obtained the following refinement of the inequality (1.2) and proved that if $p(z)$ has all zeros in $|z| \leq 1$, then

$$
\max _{|z|=1}\left|p^{\prime}(z)\right| \geq \frac{n}{2}\left\{\max _{|z|=1}|p(z)|+\min _{|z|=1}|p(z)|\right\} .
$$

This result is best possible and equality attains for a polynomial that has all zeros on $|z|=1$.

Let $D_{\alpha} p(z)$ denote the polar differentiation of the polynomial $p(z)$ of degree $n$ with respect to $\alpha \in \mathbb{C}$. Then, $D_{\alpha} p(z)=n p(z)+(\alpha-z) p^{\prime}(z)$. The polynomial $D_{\alpha} p(z)$ is of degree at most $n-1$, and it generalizes the ordinary derivative in the sense that

$$
\lim _{\alpha \rightarrow \infty}\left[\frac{D_{\alpha} p(z)}{\alpha}\right]=p^{\prime}(z)
$$

Shah [5] extended (1.2) to the polar derivative of $p(z)$ and proved that if all zeros of the polynomial $p(z)$ lie in $|z| \leq 1$, then for every $\alpha$ with $|\alpha| \geq 1$, we have

$$
\max _{|z|=1}\left|D_{\alpha} p(z)\right| \geq \frac{n}{2}(|\alpha|-1) \max _{|z|=1}|p(z)| .
$$

This result is best possible and equality holds as $p(z)=(z-1)^{n}$ with $\alpha \geq 1$.

Aziz and Rather [6] generalized (1.5) by extending (1.3) to the polar derivative of a polynomial. In fact, they proved that if all zeros of $p(z)$ lie in $|z| \leq k$, where $k \leq 1$, then for every $\alpha$ with $|\alpha| \geq k$, we get

$$
\max _{|z|=1}\left|D_{\alpha} p(z)\right| \geq \frac{n}{1+k}(|\alpha|-k) \max _{|z|=1}|p(z)| .
$$

This result is best possible and equality holds for $p(z)=(z-k)^{n}$ with $\alpha \geq k$.

In the same paper, Aziz and Rather [6] sharpened the inequality (1.5) by proving that if all the zeros of $p(z)$ lie in $|z| \leq 1$, then for every $\alpha$ with $|\alpha| \geq 1$, we would obtain

$$
\max _{|z|=1}\left|D_{\alpha} p(z)\right| \geq \frac{n}{2}\left\{(|\alpha|-1) \max _{|z|=1}|p(z)|+(|\alpha|-1) \min _{|z|=1}|p(z)|\right\} .
$$

This result is best possible and equality attains for $p(z)=(z-1)^{n}$ with $\alpha \geq 1$.

As an extension to the inequality (1.7), Jain [7] proved that if $p(z)$ has all zeros in $|z|$ $\leq 1$, then for all $\alpha_{1}, \ldots \alpha_{t} \in \mathbb{C}$ with $\left|\alpha_{1}\right| \geq 1,\left|\alpha_{2}\right| \geq 1, \ldots,\left|\alpha_{t}\right| \geq 1,(1 \leq t<n)$, we have

$$
\begin{array}{r}
\max _{|z|=1}\left|D_{\alpha_{t}} \cdots D_{\alpha_{2}} D_{\alpha_{1}} p(z)\right| \geq \frac{n(n-1) \cdots(n-t+1)}{2^{t}}[ \\
\left\{\left(\left|\alpha_{1}\right|-1\right) \cdots\left(\left|\alpha_{t}\right|-1\right)\right\} \max _{|z|=1}|p(z)|+ \\
\left.\left\{2^{t}\left(\left|\alpha_{1}\right| \cdots\left|\alpha_{t}\right|\right)-\left\{\left(\left|\alpha_{1}\right|-1\right) \cdots\left(\left|\alpha_{t}\right|-1\right)\right\}\right\} \min _{|z|=1}|p(z)|\right],
\end{array}
$$


where

$$
\begin{array}{r}
D_{\alpha_{j}} D_{\alpha_{j-1}} \cdots D_{\alpha_{1}} p(z)=p_{j}(z)= \\
(n-j+1) p_{j-1}(z)+\left(\alpha_{j}-z\right) p_{j-1^{\prime}}(z), \quad j=1,2, \cdots, t, \\
p_{0}(z)=p(z) .
\end{array}
$$

This result is best possible and equality holds as $p(z)=(z-1)^{n}$ with $\alpha_{1} \geq 1, \alpha_{2} \geq 1, \ldots$, $\alpha_{t} \geq 1$.

The following result proposes an extension to (1.8). In a precise set up, we have

Theorem 1.1. Let $p(z)$ be a polynomial of degree $n$ having all zeros in $|z| \leq k$, where $k$ $\leq 1$, then for all $\alpha_{1}, \ldots \alpha_{t} \in \mathbb{C}$ with $\left|\alpha_{1}\right| \geq k,\left|\alpha_{2}\right| \geq k, \ldots,\left|\alpha_{t}\right| \geq k,(1 \leq t<n)$,

$$
\begin{array}{r}
\max _{|z|=1}\left|D_{\alpha_{t}} \cdots D_{\alpha_{2}} D_{\alpha_{1}} p(z)\right| \geq \frac{n(n-1) \cdots(n-t+1)}{(1+k)^{t}}[ \\
\left\{\left(\left|\alpha_{1}\right|-k\right) \cdots\left(\left|\alpha_{t}\right|-k\right)\right\} \max _{|z|=1}|p(z)|+ \\
\left.\left\{(1+k)^{t}\left(\left|\alpha_{1}\right| \cdots\left|\alpha_{t}\right|\right)-\left\{\left(\left|\alpha_{1}\right|-k\right) \cdots\left(\left|\alpha_{t}\right|-k\right)\right\}\right\} k^{-n} \min _{|z|=k}|p(z)|\right] .
\end{array}
$$

This result is best possible and equality holds for $p(z)=(z-k)^{n}$ with $\alpha_{1} \geq k, \alpha_{2} \geq k, \ldots$, $\alpha_{t} \geq k$.

If we take $k=1$ in Theorem 1.1, then inequality (1.9) reduces to inequality (1.8).

If we take $t=1$ in Theorem 1.1, the following refinement of inequality (1.6) can be obtained.

Corollary 1.2. Let $p(z)$ be a polynomial of degree $n$, having all zeros in $|z| \leq k$, where $k \leq 1$, then for every $\alpha \in \mathbb{C}$ with $|\alpha| \geq k$,

$$
\max _{|z|=1}\left|D_{\alpha} p(z)\right| \geq \frac{n}{1+k}\left\{(|\alpha|-k) \max _{|z|=1}|p(z)|+(|\alpha|+1) k^{-(n-1)} \min _{|z|=k}|p(z)|\right\} .
$$

This result is best possible and equality occurs if $p(z)=(z-k)^{n}$ with $\alpha \geq k$.

If we divide both sides of the above inequality in (1.10) by $|\alpha|$ and make $|\alpha| \rightarrow \infty$, we obtain a result proved by Govil [8].

\section{Lemmas}

For proof of the theorem, the following lemmas are needed. The first lemma is due to Laguerre [9].

Lemma 2.1. If all the zeros of an nth degree polynomial $p(z)$ lie in a circular region $C$ and $w$ is any zero of $D_{\alpha} p(z)$, then at most one of the points $w$ and $\alpha$ may lie outside $C$.

Lemma 2.2. If $p(z)$ is a polynomial of degree $n$, having all zeros in the closed disk $|z|$ $\leq k, k \leq 1$, then on $|z|=1$,

$$
\left|p^{\prime}(z)\right| \geq \frac{n}{1+k}|p(z)| .
$$

This lemma is due to Govil [10].

Lemma 2.3. If $p(z)$ is a polynomial of degree $n$, having no zeros in $|z|<k, k \geq 1$, then on $|z|=1$,

$$
k\left|p^{\prime}(z)\right| \leq\left|q^{\prime}(z)\right|,
$$

where $q(z)=z^{n} \overline{p(1 / \bar{z})}$. 
The above lemma is due to Chan and Malik [11].

Lemma 2.4. If $p(z)$ is a polynomial of degree $n$, having all zeros in the closed disk $|z|$ $\leq k, k \leq 1$, then on $|z|=1$,

$$
\left|q^{\prime}(z)\right| \leq k\left|p^{\prime}(z)\right|
$$

where $q(z)=z^{n} \overline{p(1 / \bar{z})}$.

Proof. Since $p(z)$ has all its zeros in $|z| \leq k, k \leq 1$, therefore $q(z)$ has no zero in $|z|<$ $1 / k, 1 / k \geq 1$. Now applying Lemma 2.3 to the polynomial $q(z)$ and the result follows.

Lemma 2.5. If $p(z)$ is a polynomial of degree $n$, having all zeros in the closed disk $|z|$ $\leq k, k \leq 1$, then for every real or complex number $\alpha$ with $|\alpha| \geq k$ and $|z|=1$, we have

$$
\left|D_{\alpha} p(z)\right| \geq \frac{n}{1+k}(|\alpha|-k)|p(z)| .
$$

Proof. Let $q(z)=z^{n} \overline{p(1 / \bar{z})}$, then $\left|q^{\prime}(z)\right|=\left|n p(z)-z p^{\prime}(z)\right|$ on $|z|=1$. Thus, on $|z|=1$, we get

$$
\begin{aligned}
\left|D_{\alpha} p(z)\right|=\left|n p(z)+(\alpha-z) p^{\prime}(z)\right|= & \left|\alpha p^{\prime}(z)+n p(z)-z p^{\prime}(z)\right| \geq \\
& \left|\alpha p^{\prime}(z)-\right| n p(z)-z p^{\prime}(z) \mid,
\end{aligned}
$$

that implies

$$
\left|D_{\alpha} p(z)\right| \geq|\alpha|\left|p^{\prime}(z)\right|-\left|q^{\prime}(z)\right| .
$$

By combining (2.3) and (2.5), we obtain

$$
\left|D_{\alpha} p(z)\right| \geq(|\alpha|-k)\left|p^{\prime}(z)\right| .
$$

that along Lemma 2.2, yields

$$
\left|D_{\alpha} p(z)\right| \geq \frac{n}{1+k}(|\alpha|-k)|p(z)| .
$$

Lemma 2.6. If $p(z)=a_{0}+a_{1} z+\sum_{i=2}^{n} a_{i} z^{i}$ is a polynomial of degree $n$, having no zeros in $|z|<k, k \geq 1$, then

$$
\frac{k\left|a_{1}\right|}{\left|a_{0}\right|} \leq n
$$

The above lemma is due to Gardner et al. [12].

Lemma 2.7. If $p(z)=\sum_{i=0}^{n} a_{i} z^{i}$ is a polynomial of degree $n$, having all zeros in $|z| \leq k$, $k \leq 1$, then

$$
\frac{\left|a_{n-1}\right|}{\left|a_{n}\right|} \leq n k
$$

Proof. Since $p(z)$ has all zeros in $|z| \leq k, k \leq 1$, therefore

$$
q(z)=z^{n} \overline{p(1 / \bar{z})}=\overline{a_{n}}+\overline{a_{n-1}} z+\cdots+\overline{a_{1}} z^{n-1}+\overline{a_{0}} z^{n},
$$

is a polynomial of degree at most $n$, which does not vanish in $|z|<1 / k, 1 / k \geq 1$. By applying Lemma 2.6 for $q(z)$, we get 


$$
\frac{\frac{1}{k}\left|a_{n-1}\right|}{\left|a_{n}\right|} \leq \operatorname{degree}\{q(z)\} \leq n,
$$

which completes the proof.

Lemma 2.8. If $p(z)$ is a polynomial of degree $n$ having all zeros in $|z| \leq k, k \leq 1$, then for all $\alpha_{1}, \ldots \alpha_{t} \in \mathbb{C}$ with $\left|\alpha_{1}\right| \geq k,\left|\alpha_{2}\right| \geq k, \ldots,\left|\alpha_{t}\right| \geq k,(1 \leq t<n)$, and $|z|=1$ we have

$$
\begin{array}{r}
\left|D_{\alpha_{t}} \cdots D_{\alpha_{2}} D_{\alpha_{1}} p(z)\right| \geq \frac{n(n-1) \cdots(n-t+1)}{(1+k)^{t}} \times \\
\left\{\left(\left|\alpha_{1}\right|-k\right) \cdots\left(\left|\alpha_{t}\right|-k\right)\right\}|p(z)| .
\end{array}
$$

Proof. If $\left|\alpha_{j}\right|=k$ for at least one $j ; 1 \leq j \leq t$, then inequality (2.8) is trivial. Therefore, we assume that $\left|\alpha_{j}\right|>k$ for all $j ; 1 \leq j \leq t$.

In the rest, we proceed by mathematical induction. The result is true for $t=1$, by Lemma 2.5, that means if $\left|\alpha_{1}\right|>k$ then

$$
\left|D_{\alpha_{1}} p(z)\right| \geq \frac{n}{1+k}\left(\left|\alpha_{1}\right|-k\right)|p(z)| .
$$

Now for $t=2$, since $D_{\alpha_{1}} p(z)=\left(n a_{n} \alpha_{1}+a_{n-1}\right) z^{n-1}+\cdots+\left(n a_{0}+\alpha_{1} a_{1}\right)$, and $\left|\alpha_{1}\right|>k$, then $D_{\alpha_{1}} p(z)$ will be a polynomial of degree $(n-1)$. If it is not true, then the coefficient of $z^{n-1}$ must be equal to zero, which implies

$$
n a_{n} \alpha_{1}+a_{n-1}=0,
$$

i.e,

$$
\left|\alpha_{1}\right|=\frac{\left|a_{n-1}\right|}{n\left|a_{n}\right|}
$$

Applying Lemma 2.7, we get

$$
\left|\alpha_{1}\right|=\frac{\left|a_{n-1}\right|}{n\left|a_{n}\right|} \leq k
$$

But this result contradicts the fact that $\left|\alpha_{1}\right|>k$. Hence, the polynomial $D_{\alpha_{1}} p(z)$ must be of degree $(n-1)$.

On the other hand, since all the zeros of $p(z)$ lie in $|z| \leq k$, therefore by applying Lemma 2.1, all the zeros of $D_{\alpha_{1}} p(z)$ lie in $|z| \leq k$, then using Lemma 2.5 for the polynomial $D_{\alpha_{1}} p(z)$ of degree $n-1$, and $\left|\alpha_{2}\right|>k$, it concludes that

$$
\left|D_{\alpha_{2}}\left\{D_{\alpha_{1}} p(z)\right\}\right| \geq \frac{(n-1)}{1+k}\left(\left|\alpha_{2}\right|-k\right)\left|D_{\alpha_{1}} p(z)\right| .
$$

Substituting the term $D_{\alpha_{1}} p(z)$ from (2.9) in the above inequality, we obtain

$$
\left|D_{\alpha_{2}} D_{\alpha_{1}} p(z)\right| \geq \frac{n(n-1)}{(1+k)^{2}}\left(\left|\alpha_{1}\right|-k\right)\left(\left|\alpha_{2}\right|-k\right)|p(z)| .
$$

This implies result is true for $t=2$.

At this stage, we assume that the result is true for $t=s<n$; it means that for $|z|=1$, we have 


$$
\begin{array}{r}
\left|D_{\alpha_{s}} \cdots D_{\alpha_{2}} D_{\alpha_{1}} p(z)\right| \geq \frac{n(n-1) \cdots(n-s+1)}{(1+k)^{s}} \times \\
\left\{\left(\left|\alpha_{1}\right|-k\right) \cdots\left(\left|\alpha_{s}\right|-k\right)\right\}|p(z)|,
\end{array}
$$

and we will prove that the result is true for $t=s+1<n$.

According to the above procedure, using Lemmas 2.7 and 2.1, the polynomial $D_{\alpha_{2}} D_{\alpha_{1}} p(z)$ must be of degree $(n-2)$ for $\left|\alpha_{1}\right|>k,\left|\alpha_{2}\right|>k$, and has all zeros in $|z| \leq k$. One can continue that $D_{\alpha_{s}} \cdots D_{\alpha_{2}} D_{\alpha_{1}} p(z)$ will be a polynomial of degree $(n-s)$ for all $\alpha_{1}, \ldots \alpha_{s} \in \mathbb{C}$ with $\left|\alpha_{1}\right| \geq k,\left|\alpha_{2}\right| \geq k, \ldots,\left|\alpha_{s}\right| \geq k$, $(s<n)$, and has all zeros in $|z| \leq k$. Therefore, for $\left|\alpha_{s+1}\right|>k$, by applying Lemma 2.5 to $D_{\alpha_{s}} \cdots D_{\alpha_{2}} D_{\alpha_{1}} p(z)$, we get

$$
\left|D_{\alpha_{s+1}}\left\{D_{\alpha_{s}} \cdots D_{\alpha_{2}} D_{\alpha_{1}} p(z)\right\}\right| \geq \frac{(n-s)}{1+k}\left(\left|\alpha_{s+1}\right|-k\right)\left|D_{\alpha_{s}} \cdots D_{\alpha_{2}} D_{\alpha_{1}} p(z)\right| .
$$

By combining the terms (2.10) and (2.11), we obtain

$$
\begin{array}{r}
\left|D_{\alpha_{s+1}} D_{\alpha_{s}} \cdots D_{\alpha_{2}} D_{\alpha_{1}} p(z)\right| \geq \frac{n(n-1) \cdots(n-s)}{(1+k)^{s+1}} \times \\
\left\{\left(\left|\alpha_{1}\right|-k\right) \cdots\left(\left|\alpha_{s+1}\right|-k\right)\right\}|p(z)| .
\end{array}
$$

This implies that the result is true for $t=s+1$. The proof is complete.

Lemma 2.9. If $p(z)=\sum_{i=0}^{n} a_{i} z^{i}$ is a polynomial of degree $n, p(z) \neq 0$ in $|z|<k$, then $m$ $<|p(z)|$ for $|z|<k$, and in particular $m<\left|a_{0}\right|$, where $m=\min _{|z|=k}|p(z)|$.

The above lemma is due to Gardner et al. [13].

Lemma 2.10. If $p(z)=\sum_{i=0}^{n} a_{i} z^{i}$ is a polynomial of degree $n$ having all zeros in $|z| \leq$ $k$, then

$$
m \leq k^{n}\left|a_{n}\right|,
$$

where $m=\min _{|z|=k}|p(z)|$.

Proof. If $k=0$, then inequality (2.12) is trivial. Now we suppose that $k>0$. Since the polynomial $p(z)=\sum_{i=0}^{n} a_{i} z^{i}$ has all zeros in $|z| \leq k$, the polynomial $q(z)=z^{n} p(1 / z)=$ $a_{n}+\ldots+a_{0} z^{n}$ has no zero in $|z|<\frac{1}{k}$. Thus, by applying Lemma 2.9 for the polynomial $q(z)$, we get

$$
\min _{|z|=\frac{1}{k}}|q(z)|<\left|a_{n}\right|
$$

Since $\min _{|z|=\frac{1}{k}}|q(z)|=\frac{1}{k^{n}} \min _{|z|=k}|p(z)|$, (2.13) implies that $\frac{m}{k^{n}}<\left|a_{n}\right|$.

\section{Proof of the theorem}

Proof of Theorem 1.1. Let $m=\min _{|z|=k}|p(z)|$. If $p(z)$ has a zero on $|z|=k$, then $m=$ 0 and the result follows from Lemma 2.8. Henceforth, we suppose that all the zeros of $p(z)$ lie in $|z|<k$, so that $m>0$. Now $m \leq|p(z)|$ for $|z|=k$, therefore if $\lambda$ is any real or complex number such that $|\lambda|<1$, then $\left|\lambda m\left(\frac{z}{k}\right)^{n}\right|<|p(z)|$ for $|z|=k$. Since all zeros of $p(z)$ lie in $|z|<k$, by Rouche's theorem we can deduce that all zeros of the polynomial $G(z)=p(z)-\lambda m\left(\frac{z}{k}\right)^{n}$ lie in $|z|<k$. Also it follows from Lemma 2.10, that 
$G(z)=p(z)-\lambda\left(\frac{m}{k^{n}}\right) z^{n}$, hence the polynomial $G(z)=p(z)-\lambda\left(\frac{m}{k^{n}}\right) z^{n}$ is of degree $n$. Now we can apply Lemma 2.8 for the polynomial $G(z)$ of degree $n$ which has all zeros in $|z| \leq k$. This implies that for all $\alpha_{1}, \ldots \alpha_{t} \in \mathbb{C}$ with $\left|\alpha_{1}\right| \geq k,\left|\alpha_{2}\right| \geq k, \ldots,\left|\alpha_{t}\right| \geq k,(t$ $<n)$, on $|z|=1$,

$$
\begin{array}{r}
\left|D_{\alpha_{t}} \cdots D_{\alpha_{2}} D_{\alpha_{1}} G(z)\right| \geq \frac{n(n-1) \cdots(n-t+1)}{(1+k)^{t}} \times \\
\left\{\left(\left|\alpha_{1}\right|-k\right) \cdots\left(\left|\alpha_{t}\right|-k\right)\right\}|G(z)| .
\end{array}
$$

Equivalently

$$
\begin{gathered}
\left|D_{\alpha_{t}} \cdots D_{\alpha_{2}} D_{\alpha_{1}} p(z)-\lambda \frac{m}{k^{n}}\left\{n(n-1) \cdots(n-t+1) \alpha_{1} \alpha_{2} \cdots \alpha_{1}\right\} z^{n-t}\right| \geq \\
\frac{n(n-1) \cdots(n-t+1)}{(1+k)^{t}}\left\{\left(\left|\alpha_{1}\right|-k\right) \cdots\left(\left|\alpha_{t}\right|-k\right)\right\}\left|p(z)-\lambda m\left(\frac{z}{k}\right)^{n}\right| .
\end{gathered}
$$

But by Lemma 2.1, the polynomial $T(z)=D_{\alpha_{t}} \cdots D_{\alpha_{2}} D_{\alpha_{1}} G(z)$ has all zeros in $|z| \leq k$. That is,

$$
T(z)=D_{\alpha_{t}} \cdots D_{\alpha_{2}} D_{\alpha_{1}} G(z) \neq 0, \text { for }|z|>k .
$$

Then, substituting $G(z)$ in the above, we conclude that for every $\lambda$ with $|\lambda|<1$, and $\mid$ $z \mid>k$,

$$
\begin{array}{r}
T(z)=D_{\alpha_{t}} \cdots D_{\alpha_{2}} D_{\alpha_{1}} p(z)- \\
\lambda \frac{m}{k^{n}}\left\{n(n-1) \cdots(n-t+1) \alpha_{1} \alpha_{2} \cdots \alpha_{t}\right\} z^{n-t} \neq 0 .
\end{array}
$$

Thus, for $|z|>k$,

$$
\left|D_{\alpha_{t}} \cdots D_{\alpha_{2}} D_{\alpha_{1}} p(z)\right| \geq \frac{m}{k^{n}}\left\{n(n-1) \cdots(n-t+1)\left|\alpha_{1}\right|\left|\alpha_{2}\right| \cdots\left|\alpha_{t}\right|\right\}\left|z^{n-t}\right| .
$$

If the inequality (3.3) is not true, then there is a point $z=z_{0}$ with $\left|z_{0}\right|>k$ such that

$$
\left|D_{\alpha_{t}} \cdots D_{\alpha_{2}} D_{\alpha_{1}} p\left(z_{0}\right)\right|<\frac{m}{k^{n}}\left\{n(n-1) \cdots(n-t+1)\left|\alpha_{1}\right|\left|\alpha_{2}\right| \cdots\left|\alpha_{t}\right|\right\}\left|z_{0}^{n-t}\right| .
$$

Now take

$$
\lambda=\frac{D_{\alpha_{t}} \cdots D_{\alpha_{2}} D_{\alpha_{1}} p\left(z_{0}\right)}{\frac{m}{k^{n}}\left\{n(n-1) \cdots(n-t+1) \alpha_{1} \alpha_{2} \cdots \alpha_{t}\right\} z_{0}^{n-t}},
$$

then $|\lambda|<1$ and with this choice of $\lambda$, we have, $T\left(z_{0}\right)=0$ for $\left|z_{0}\right|>k$, from (3.2). But it contradicts the fact that $T(z) \neq 0$ for $|z|>k$. Hence, for $|z|>k$, we have

$$
\left|D_{\alpha_{t}} \cdots D_{\alpha_{2}} D_{\alpha_{1}} p(z)\right| \geq \frac{m}{k^{n}}\left\{n(n-1) \cdots(n-t+1)\left|\alpha_{1}\right|\left|\alpha_{2}\right| \cdots\left|\alpha_{t}\right|\right\}\left|z^{n-t}\right| .
$$

Taking a relevant choice of argument of $\lambda$, $\arg$ $\lambda=\arg \left\{D_{\alpha_{t}} \cdots D_{\alpha_{2}} D_{\alpha_{1}} p(z)\right\}-\arg \left\{\alpha_{1} \alpha_{2} \cdots \alpha_{t} z^{n-t}\right\}$, we have 


$$
\begin{array}{r}
\mid D_{\alpha_{t}} \cdots D_{\alpha_{2}} D_{\alpha_{1}} p(z)- \\
\lambda \frac{m}{k^{n}}\left\{n(n-1) \cdots(n-t+1) \alpha_{1} \alpha_{2} \cdots \alpha_{t}\right\} z^{n-t} \mid= \\
\mid D_{\alpha_{t}} \cdots D_{\alpha_{2}} D_{\alpha_{1}} p(z)- \\
|\lambda| \frac{m}{k^{n}}\left\{n(n-1) \cdots(n-t+1)\left|\alpha_{1}\right|\left|\alpha_{2}\right| \cdots\left|\alpha_{t}\right|\right\}\left|z^{n-t}\right|,
\end{array}
$$

where $|z|=1$.

Therefore, we can rewrite (3.1) as

$$
\begin{array}{r}
\left|D_{\alpha_{t}} \cdots D_{\alpha_{2}} D_{\alpha_{1}} p(z)\right|- \\
|\lambda| \frac{m}{k^{n}}\left\{n(n-1) \cdots(n-t+1)\left|\alpha_{1}\right|\left|\alpha_{2}\right| \cdots\left|\alpha_{t}\right|\right\} z^{n-t} \mid \geq \\
\frac{n(n-1) \cdots(n-t+1)}{(1+k)^{t}}\left\{\left(\left|\alpha_{1}\right|-k\right) \cdots\left(\left|\alpha_{t}\right|-k\right)\right\}\left(p(z)-|\lambda| \frac{m}{k^{n}}|z|^{n}\right),
\end{array}
$$

where $|z|=1$.

In an equivalent way

$$
\begin{gathered}
\left|D_{\alpha_{t}} \cdots D_{\alpha_{2}} D_{\alpha_{1}} p(z)\right| \geq \frac{n(n-1) \cdots(n-t+1)}{(1+k)^{t}}[ \\
\left\{\left(\left|\alpha_{1}\right|-k\right) \cdots\left(\left|\alpha_{t}\right|-k\right)|p(z)|\right\}+ \\
\left.|\lambda|\left\{(1+k)^{t}\left(\left|\alpha_{1}\right|\left|\alpha_{2}\right| \cdots\left|\alpha_{t}\right|\right)-\left\{\left(\left|\alpha_{1}\right|-k\right) \cdots\left(\left|\alpha_{t}\right|-k\right)\right\}\right\} \frac{m}{k^{n}}\right] .
\end{gathered}
$$

\section{Making $|\lambda| \rightarrow 1$, Theorem 1.1 follows.}

\section{Acknowledgements}

The author is grateful to the referees, for the careful reading of the paper and for the helpful suggestions and comments. This research was supported by Shahrood University of Technology.

\section{Competing interests}

The author declares that they have no competing interests.

\section{Received: 25 April 2011 Accepted: 10 November 2011 Published: 10 November 2011}

\section{References}

1. Bernstein, S: Leons sur les Proprits Extrmales et la Meilleure Approximation des Fonctions Analytiques dune Variable relle. Gauthier Villars, Paris (1926)

2. Turan, P: Uber die ableitung von Polynomen. Compositio Math. 7, 89-95 (1939)

3. Malik, MA: On the derivative of a polynomial. J Lond Math Soc. 1, 57-60 (1969). doi:10.1112/Jms/s2-1.1.57

4. Aziz, A, Dawood, QM: Inequalities for a polynomial and its derivative. J Approx Theory. 54, 306-313 (1988). doi:10.1016/ 0021-9045(88)90006-8

5. Shah, WM: A generalization of a theorem of Paul Turan. J Ramanujan Math Soc. 1, 67-72 (1996)

6. Aziz, A, Rather, NA: A refinement of a theorem of Paul Turan concerning polynomials. Math Ineq Appl. 1, 231-238 (1998)

7. Jain, VK: Generalization of an inequality involving maximum moduli of a polynomial and its polar derivative. Bull Math Soc Sci Math Roum Tome. 98, 67-74 (2007)

8. Govil, NK: Some inequalities for derivative of polynomials. J Approx Theory. 66, $29-35$ (1991). doi:10.1016/0021-9045(91) $90052-C$

9. Laquerre, E: OEuvres. Vol. pp. 48-66. Chelsea, New York, 21.

10. Govil, NK: On the derivative of a polynomial. Proc Am Math Soc. 41, 543-546 (1973). doi:10.1090/S0002-9939-19730325932-8

11. Chan, TN, Malik, MA: On Erdoös-Lax Theorem. Proc. Indian Acad Sci. 92, 191-193 (1983). doi:10.1007/BF02876763

12. Gardner, RB, Govil, NK, Weems, A: Some results concerning rate of growth of polynomials. East J on Approx. 10, 301-312 (2004)

13. Gardner, RB, Govil, NK, Musukula, SR: Rate of growth of polynomials not vanishing inside a circle. J Inequal Pure Appl Math. 6, 1-9 (2005) 
doi:10.1186/1029-242X-2011-111

Cite this article as: Zireh: On the maximum modulus of a polynomial and its polar derivative. Journal of Inequalities and Applications 2011 2011:111.

Submit your manuscript to a SpringerOpen ${ }^{\odot}$ journal and benefit from:

- Convenient online submission

- Rigorous peer review

- Immediate publication on acceptance

- Open access: articles freely available online

- High visibility within the field

- Retaining the copyright to your article

Submit your next manuscript at $\gg$ springeropen.com 